\section{Virtual arrays}

\section{JOHN R. VOKEY \\ University of Lethbridge, Lethbridge, Alberta, Canada}

Virtual arrays are common to the programming languages of many mini and mainframe computer systems. They allow the programmer to write computer programs containing numerical arrays of virtually unlimited capacity. In fact, for many of these systems, the only limitation on the size of arrays that may be used is the capacity of the disk operating system. In contrast, most BASIC programming languages available for microcomputers, including the floating-point version for the Apple // (Applesoft BASIC), limit the size of arrays to the amount of available on-board memory, which, after allowing for the disk operating system, file buffers, and system memory (zero, input buffer, and stack pages; page three of operating system vectors; and the primary display pages), disappears fairly rapidly for even relatively moderate data bases-and that is before memory is set aside for the application program itself.

In this article a patch to Applesoft BASIC is described that provides for both real and integer virtual arrays. The patch will function on any Apple // (or compatible) computer capable of using the ProDOS BASIC.SYSTEM. Aside from the initial dimensioning of the virtual arrays, most Applesoft BASIC programs should execute without modification, typically requiring less user memory than before, but allowing for virtual arrays of up to 16 megabytes (MB) each. Moreover, nothing about the patch affects the use of normal (nonvirtual) arrays. Only those arrays that are too large for main memory (or for which automatic storage to disk is desired) need be declared virtual; all others may be dimensioned and used as usual.

\section{Command Definition and Syntax}

The virtual array patch to Applesoft BASIC adds three new commands to the language, interfaced to Applesoft BASIC via the ampersand vector.

\& DEF pathname, varname $(\mathrm{I}, \mathrm{J}, \ldots, \mathrm{N})$. The \& DEF statement defines the specified real or integer array as virtual and creates a virtual array file under the specified ProDOS path name. For example, the statement

\section{\& DEF"/RAM/EXAMPLE",X(100,100),}

in either immediate or deferred mode, will dimension the real array, X, to contain 50 kilobytes $(K)(101 \times 101$ elements, with 5 bytes per element) of data, and will create the corresponding $50 \mathrm{~K}$ file on the "/RAM" volume un-

Preparation of this article was supported in part by an operating grant from the Natural Sciences and Engineering Research Council of Canada to the author. Requests for the virtual array software should be addressed to J. R. Vokey, Department of Psychology, University of Lethbridge, Lethbridge, Alberta T1K 3M4, Canada. der the file name "EXAMPLE." From that point on, the array may be used in exactly the same fashion as any normal (nonvirtual) real array in Applesoft BASIC.

Any string expression that evaluates to a legal ProDOS path name may be used as the virtual array file name in the \& DEF statement. If a virtual array file of the specified name already exists, the patch will retrieve the data from it, rather than create a new file. In this way, virtual arrays may be shared across programs, or even within the same program by array variable "aliases." For real arrays, the virtual array data are stored in Apple's 5-byte, packed floating-point format, whereas integer virtual array data require 2 bytes per element. In either case, the data are stored as a random-access file (arbitrarily labeled with the ProDOS typeless file code, $\$ 00$ ), resulting in a more space-efficient and more rapidly accessed file structure than the more usual sequential text file.

Any array declaration that would be legal in an Applesoft BASIC real or integer DIMension statement may be used to dimension the array in the \& DEF statement, with the addition that array sizes greater than $64 \mathrm{~K}$ (the theoretical maximum in Applesoft BASIC), up to $16 \mathrm{MB}$ (the limit to ProDOS files), may be declared as virtual. ${ }^{1}$

\& STORE varname $(0,0, \ldots, 0)$. The virtual array patch to Applesoft BASIC works by swapping 512-byte blocks of array data between the array file and memory as needed, updating the file in the process. However, because the block of array data currently in memory is not updated in the file unless another block is swapped into memory, any changes to the last block accessed would not be saved to the file. The \& STORE command, in either immediate or deferred mode, may be used to ensure that any changes to the data in the block of the specified virtual array currently in memory are stored to the file. To continue the earlier example, the command

$$
\text { \& STORE X(0,0) }
$$

could be used at the end of a program (or before chaining or loading another program) to ensure that any changes to the last block accessed by the program were stored to the virtual array file. The values (expression or numerical) of the array indices in the \& STORE statement are immaterial, but they are evaluated and therefore must be within the bounds declared for the array.

\& CLEAR \{var[,var]\}. The \& CLEAR command, in either immediate or deferred mode, clears the specified variable(s) of any type, simple or array, from memory, freeing the memory formerly occupied for other data. The algorithm for the command is adapted from the "dispose" utility developed by Kaner and Vokey (1985), in which further details on its use may be found.

\section{The Virtual Array Patch}

The virtual array patch to Applesoft BASIC is written in 6502 assembler language. When initially executed, it 
relocates itself into six free pages of high memory and interfaces itself to Applesoft BASIC through the ampersand and CHRGET vectors (see Kaner \& Vokey, 1982, for details on these methods of adding patches to Applesoft BASIC). The patch automatically daisy chains the ampersand vector so that other Applesoft BASIC ampersand utilities currently patched to the system will continue to function. Once installed on the system, the patch will remain available for use until an Applesoft BASIC or BASIC.SYSTEM cold start.

The CHRGET interface is used to intercept all array calls by the Applesoft BASIC interpreter and allows the patch to swap in the required virtual array data as needed. When a block of virtual array data is swapped into memory, the array element requested by the interpreter is loaded as the middle element of the block. This procedure allows for both forward and backward stepping from that point of about 50 real array elements and over 125 integer array elements before a new block of array data needs to be swapped into memory.

Programs that use virtual arrays will execute more slowly than their nonvirtual array equivalents. The extent to which they execute more slowly depends on the storage medium for the virtual array data and on how frequently blocks of array data are swapped into memory. RAM disks and hard disks are, because of their superior rates of data transfer, the preferred storage media for virtual arrays, and any slowing of program execution is just barely noticeable, even with frequent swapping of data blocks. With floppy diskettes, however, the time required to swap data blocks between the disk file and memory is on the order of seconds so that maximum efficiency is achieved by making maximum use of each virtual array block while it is in memory. To this end, it is important to recognize that the data for Applesoft BASIC arrays (and, hence, virtual arrays) are ordered such that the first index changes fastest through to the last index, which changes slowest, contrary to convention. Thus a statement such as "FOR I = 1 TO 100: FOR J = 1 TO 100: X(I,J) $=0$ : NEXT J,I" will access the array data out of order and, consequently, require more frequent swapping of data blocks than would the logically equivalent statement "FOR I = 1 TO 100: FOR J = 1 TO 100: X(J,I) = 0: NEXT J,I."

\section{Error Messages}

The virtual array patch supports all Applesoft BASIC and ProDOS BASIC.SYSTEM error messages and the error trapping provided by the Applesoft BASIC " $\mathrm{ON}$ ERR GOTO ..." command.

\section{Availability}

The virtual array patch to Applesoft BASIC is available as a package, including the object code and commented source code, on a ProDOS floppy diskette, and may be obtained free of charge by writing the author and enclosing an Apple-compatible floppy diskette.

\section{REFERENCES}

Kaner, H. C., \& VoKeY, J. R. (1982). Modifying Apple's floating point BASIC: An \& interpreter without the \&. Compute!, 24, 146-152. KANER, H. C., \& VokeY, J. R. (1985). Subroutine master. Nibble, 6(11), 46-75.

\section{NOTE}

1. The ProDOS limit of $16 \mathrm{MB}$ per virtual array file represents the theoretical upper limit. Most external storage devices typically are limited to considerably less data. A single floppy diskette, for example, is limited under ProDOS to 280 blocks (140K). Even this limited capacity of floppy diskettes, however, allows for virtual arrays of over 28,000 real or 71,000 integer elements, which is considerably more than that allowed for in Applesoft BASIC.

(Revision accepted for publication November 21, 1986.) 